\title{
Ab Initio Study of He Migrations in Fcc Au-Ag Alloys
}

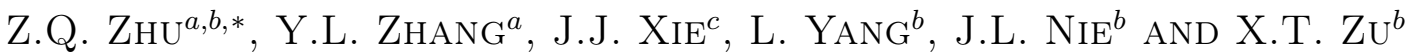 \\ ${ }^{a}$ School of Physics and Telecommunication Engineering, Zhoukou Normal University, Zhoukou 466001, China \\ ${ }^{b}$ School of Physical Electronics, University of Electronic Science and Technology of China, Chengdu 610054, China \\ ${ }^{c}$ School of Mechanical and Electrical Engineering, Zhoukou Normal University, Zhoukou 466001, China
}

(Received October 22, 2015; revised version March 25, 2016; in final form April 4, 2016)

\begin{abstract}
$A b$ initio calculations based on the density functional theory have been performed to investigate the migrations of interstitial helium $(\mathrm{He})$ atoms in $\mathrm{Au}-\mathrm{Ag}$ alloys with two different mass ratios $\left(\mathrm{Au}_{3} \mathrm{Ag}_{2}\right.$ and $\left.\mathrm{AuAg}\right)$. The results show that the migration mechanisms of He atoms mainly depend on the crystal structures of alloys, and their migration energy barriers are affected by the migration paths in $\mathrm{Au}-\mathrm{Ag}$ alloys. He interstitials preferentially occupied the most stable sites, but it is difficult for He interstitials to migrate to nearest most stable sites via second stable positions at room temperature. When He atom is at the tetrahedral position which has higher formation energy, it possibly migrates to nearest tetrahedral positions directly for AuAg alloy. In addition, comparing the migration of He defects in the two alloys, we found that the properties of migration energy and relative stability of He atoms probably slightly depend on the mass-density of $\mathrm{Au}-\mathrm{Ag}$ alloys.
\end{abstract}

DOI: 10.12693/APhysPolA.129.1151

PACS/topics: 66.30.J-, 81.05.Bx, 71.15.Mb

\section{Introduction}

The radioactive metal plutonium $(\mathrm{Pu})$ can release a large number of helium (He) atoms, forming selfradiation damage ageing [1]. He may easily precipitate into clusters or bubbles because of their very low solubility and high mobility, leading to degradation of the mechanical properties of $\mathrm{Pu}[2-5]$. In irradiation damage field, establishing the behavior and information of $\mathrm{He}$ in $\mathrm{Pu}$ on the atomic scale is the foundation of obtaining the impact of mechanical properties of $\mathrm{Pu}$ by $\mathrm{He}$ on larger scale [6]. Pu has as many as six established phases $[7,8]$, the fcc- $\mathrm{Pu}(\delta-\mathrm{Pu})$ is of greatest interest, because with adding a small amount of gallium or aluminium it owns the best mechanical and metallurgical properties in its stabilized form $[9,10]$. However, it is very difficult to investigate the He behavior in $\delta$ - $\mathrm{Pu}$ by experiment, because $\delta$-Pu is active and unstable at room temperature. It has been a significant development to search a replacement material of $\delta-\mathrm{Pu}$ at room temperature, which can be used to study the effects of radiation damage on mechanical properties of $\delta$-Pu indirectly. Considering crystal structures and mass-densities, Stevens et al. applied gold $(\mathrm{Au})$-silver $(\mathrm{Ag})$ alloys to replace $\delta$-Pu [11], they investigated the nucleation and growth of helium-associated defects in $\mathrm{Au}-\mathrm{Ag}$ alloys using transmission electron microscopy and found that some bubbles were not uniformly distributed, but there was no conclusive evidence to suggest that the He irradiation resulted in He bubble formation in $\mathrm{Au}-\mathrm{Ag}$ surrogate. Thomé et al. [12] investigated the radiation defects induced by He implantation in gold-based alloy $\left(\mathrm{Au}_{3} \mathrm{Ag}_{2}\right.$ alloy) using positron anni-

*corresponding author; e-mail: ziqzhu@163.com hilation spectroscopy. Their results indicated that the high-internal stresses generated by the formation of helium bubbles in the material would be the origin of the mechanical degradation in helium-implanted metallic alloys. In our previous work [13], $\mathrm{Au}-\mathrm{Ag}$ alloy systems of 60 at.\% $\mathrm{Au}$ and 40 at.\% $\mathrm{Ag}\left(\mathrm{Au}_{3} \mathrm{Ag}_{2}\right)$ and 50 at.\% $\mathrm{Au}$ and 50 at.\% $\mathrm{Ag}(\mathrm{AuAg})$ was chosen as a suitable surrogate. Our results suggested that the substitutional $\mathrm{He}$ defects are the most stable configurations in both the alloys, and the octahedral He interstitials are energetically more favorable than the tetrahedral interstitials and that the properties of He defects slightly depend on the massdensity of $\mathrm{Au}-\mathrm{Ag}$ alloys. In the present work, we have investigated the migrations of $\mathrm{He}$ atoms in $\mathrm{Au}-\mathrm{Ag}$ alloys with $a b$ initio calculations based on density functional theory (DFT).

\section{Methodology}

In order to further understand the behavior of $\mathrm{He}$ atoms in $\mathrm{Au}-\mathrm{Ag}$ alloys, the migration behavior of $\mathrm{He}$ atoms have been discussed on the basis of the research of relative stability of He defect. The climbing image nudged elastic band (CI-NEB) method [14, 15] implanted in the VASP code was used to investigate the migration mechanisms and energy barriers of He atoms diffusions in $\mathrm{Au}-\mathrm{Ag}$ alloys at a supercell with a fixed volume condition. The version number of VASP code is 4.6. This method is used to find a saddle point and a minimum energy migration path between the initial and final states of a transition, which constructs a number of intermediate images along the energy path [16]. A spring interaction between adjacent images is added to ensure continuity of the path. The transition state corresponding to the highest energy point along this path ascends. The migration energy barrier is the difference of the total energy between this transition state and the nearby local minima 
from the initial state to the final state of the path. The forces on all the atoms in each image of the CI-NEB chain converge to $0.01 \mathrm{eV}$.

\section{Results}

\subsection{The migration He defect in $\mathrm{Au}_{3} \mathrm{Ag}_{2}$ alloy}

In our previous work [13], the $\mathrm{Au}_{3} \mathrm{Ag}_{2}$ alloy includes four octahedral interstitials, three tetrahedral interstitials, and five substitutional sites. The migration of substitutional $\mathrm{He}$ are not studied in this paper, because of the huge calculating amount and the bad energy convergence. Therefore, interstitial sites are calculated only. These interstitial defect configurations are shown in Fig. 1, and are denoted as octa-1, octa-2, octa-3, and octa- 4 for octahedral He interstitials, tetra-1, tetra- 2 , and tetra-3 for tetrahedral He interstitials. The formation energies (in eV) for the octahedral He $\left(E_{\text {octa }}^{\mathrm{f}}\right)$ and the tetrahedral He $\left(E_{\text {tetra }}^{\mathrm{f}}\right)$ interstitials are shown in Table I. The octa- $1 \mathrm{He}$ are not only the most stable interstitials configurations but also the most stable octahedral interstitials configurations, and the tetra- 3 He are the most stable tetrahedral interstitials configurations. He interstitials prefer to occupy octa-1, because of the lowest relative formation energy and the most stable interstitials configurations. When octa-1 positions are occupied, whether He atoms occupy tetra-3 sites with higher formation energy? In order to solve this question, the CI-NEB method implanted in the VASP code was used to determine the migration mechanisms and energy barriers of He atoms, and the migration path of an He atom from octa- 1 to tetra-3 illustrated in Fig. 2 was considered. The migration energy path is shown in Fig. 3. The 80-atom supercell with $5 \times 2 \times 2$ fcc unit cells are used for migration.

TABLE I

Formation energies (in eV) for the octahedral He $\left(E_{\text {octa }}^{\mathrm{f}}\right)$ and the tetrahedral He $\left(E_{\text {tetra }}^{\mathrm{f}}\right)$ interstitials in a fcc$\mathrm{Au}_{3} \mathrm{Ag}_{2}$ alloy. The formation energy calculations were carried out in a 180-atom supercell with $9 \mathrm{k}$-points and a 80 -atom supercell with 32 k-points. Four octahedral and three tetrahedral interstitials He configurations were considered, as illustrated in Fig. 1 [13].

\begin{tabular}{c|c|c|c|c}
\hline \hline \multirow{3}{*}{ configuration } & \multicolumn{2}{|c|}{$\begin{array}{c}5 \times 3 \times 3 \\
(180 \text { atoms })\end{array}$} & \multicolumn{2}{c}{$\begin{array}{c}5 \times 2 \times 2 \\
(80 \text { atoms })\end{array}$} \\
\cline { 2 - 5 } & $E_{\text {octa }}^{\mathrm{f}}$ & $E_{\text {tetra }}^{\mathrm{f}}$ & $E_{\text {octa }}^{\mathrm{f}}$ & $E_{\text {tetra }}^{\mathrm{f}}$ \\
\hline 1 & 2.94 & 3.49 & 2.98 & 3.62 \\
2 & 3.42 & 3.28 & 3.41 & 3.37 \\
3 & 3.40 & 3.22 & 3.39 & 3.30 \\
4 & 3.27 & & 3.32 & \\
\hline
\end{tabular}

Figure 3 illustrates that the migration energy from octa- 1 to tetra- 3 is $0.32 \mathrm{eV}$, consistent with the formation energy difference between octa- 1 and tetra-3. If a tetra- 3 He atom migrates to octa- 1 site, there are no migration energy barriers, which means that a less stable (a)

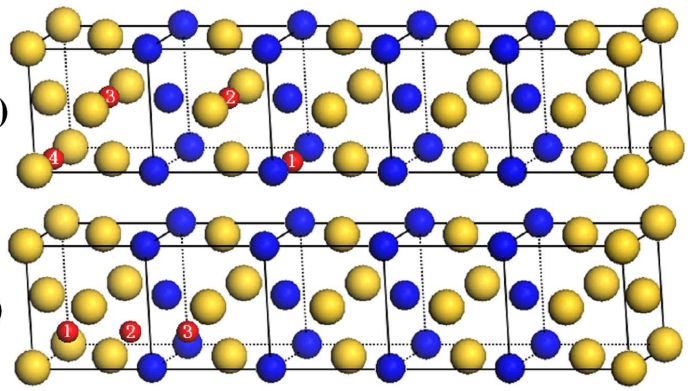

Fig. 1. Configurations of the He defects in an $\mathrm{Au}_{3} \mathrm{Ag}_{2}$ alloy: (a) four different octahedral sites, and (b) three different tetrahedral sites, where $\mathrm{Au}$ atoms are shown by the biggest golden spheres, $\mathrm{Ag}$ atoms medium blue spheres and He atoms smallest red spheres. The red spheres marked " $1,2,3,4$ " in (a) correspond to octa-1, octa- 2 , octa- 3 and octa- 4 interstitials, " $1,2,3$ " in (b) the tetra-1, tetra- 2 and tetra- 3 interstitial sites.

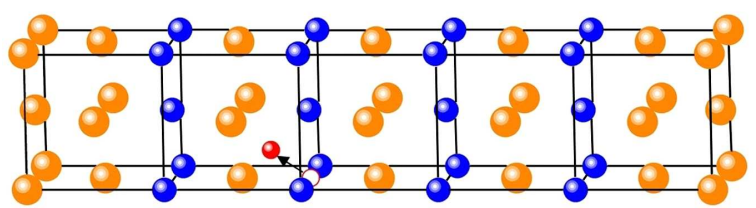

Fig. 2. Migration path of a He atom from octa-1 to tetra-3 in an $\mathrm{Au}_{3} \mathrm{Ag}_{2}$ alloy. $\mathrm{Au}$ atoms are shown by the biggest golden spheres, $\mathrm{Ag}$ atoms medium blue spheres. The smallest hollow red sphere represents the initial position of a He atom, while the smallest solid red sphere the final position, respectively. The arrow indicates the direction of the pathway of He. It is of interest to note that in contrast with Fig. 1b, the position of He atom is also tetra- 3 . In fact, the both tetra- 3 positions were considered on the calculation of formation energy.

tetra-3 He atom will automatically migrate to the stable octa-1 position. These results obtained above suggest that He will stabilize in the octa- 1 site and it is almost impossible to thermally migrate to octa-3 position at room temperature, because of higher migration energy of $0.32 \mathrm{eV}$. In fact, the tetra- 3 site is saddle point of the migration path of octa-1-tetra-3-octa-1. This result is similar to that of the He defects migration in hcp-Sc by the path of tetra-octa-tetra [17]. We demonstrated that the migration energy of tetrahedral interstitial He atom migrating to octahedral site equals to the formation energy difference between tetrahedral $\mathrm{He}$ and octahedral $\mathrm{He}$ in hcp-Sc. This result is also analogous to that of the He defects migrate in fcc-Al by the path of octa-tetra-octa. Whitmore [18] illustrated that migration energy of octahedral interstitial $\mathrm{He}$ atom to tetrahedral site equals to the formation energy difference between octahedral He and tetrahedral He in fcc-Al.

\subsection{The migration He defect in AuAg alloy}

We also calculated the stability of $\mathrm{He}$ atoms in $\mathrm{AuAg}$ alloy, in order to understand the effect of mass density on 


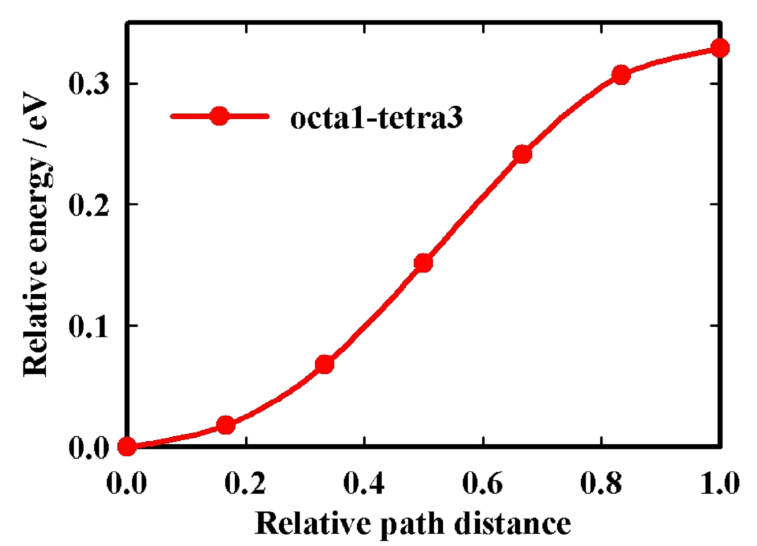

Fig. 3. Migration energy path of a $\mathrm{He}$ atom from octa- 1 to tetra-3 in an $\mathrm{Au}_{3} \mathrm{Ag}_{2}$ alloy, and the corresponding migration path shown in Fig. 2.

the He behavior in $\mathrm{Au}-\mathrm{Ag}$ alloys [13]. The AuAg alloy includes two octahedral interstitials and one tetrahedral interstitial. These defect configurations are shown in Fig. 4, and are denoted as octa- 1 and octa-2 for octahedral He interstitials. The formation energies (in $\mathrm{eV}$ ) for the octahedral He $\left(E_{\text {octa }}^{\mathrm{f}}\right)$ and the tetrahedral He $\left(E_{\text {tetra }}^{\mathrm{f}}\right)$ interstitials are shown in Table II. The octa-2 He are not only the most stable interstitials configurations but also the most stable octahedral interstitials configurations, and the stability of tetra He fall in between the octa- $2 \mathrm{He}$ and octa- $1 \mathrm{He}$, that is to say, the stabilities of interstitial configuration follow the order: octa- $2>$ tetra $>$ octa- 1 . He interstitials prefer to occupy octa-2, because of the lowest relative formation energy and the most stable interstitials configurations. When octa-2 positions are occupied, whether He atoms migrate to other octa-2 via octa-1 sites? In order to solve this doubt and understand the behavior of He in AuAg alloy in depth, the CI-NEB method implanted in the VASP code was used to determine the migration mechanisms and energy barriers of He atoms, and the migration paths of an He atom from octa- 2 to octa-1 as illustrated in Fig. 5 were considered. We also considered the migration between two neighbor tetra sites as illustrated in Fig. 5. The migration energy paths are shown in Fig. 6. The 108-atom supercell with $3 \times 3 \times 3$ fcc unit cells are used for migration.

TABLE II

Formation energies (in eV) for the octahedral He $\left(E_{\text {octa }}^{\mathrm{f}}\right)$ and the tetrahedral He $\left(E_{\text {tetra }}^{\mathrm{f}}\right)$ interstitials in a fcc-AuAg alloy. The calculations were carried out in a 108-atom supercell with 27 k-points. Two octahedral and one tetrahedral He interstitials were considered, as illustrated in Fig. 4 [13].

\begin{tabular}{c|c|c}
\hline \hline configuration & $E_{\text {octa }}^{\mathrm{f}}$ & $E_{\text {tetra }}^{\mathrm{f}}$ \\
\hline 1 & 3.53 & 3.28 \\
2 & 3.01 &
\end{tabular}

The blue curve in Fig. 6 demonstrated that the migration energy from octa- 2 to octa- 1 is $0.52 \mathrm{eV}$, consistent (a)

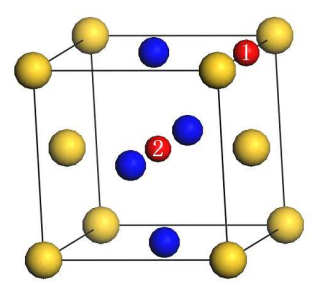

(b)

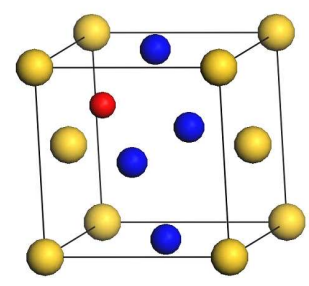

Fig. 4. Configurations of the He defects in an AuAg alloy: (a) two different octahedral sites, and (b) tetrahedral site, where $\mathrm{Au}$ atoms are shown by the biggest golden spheres, $\mathrm{Ag}$ atoms medium blue spheres and $\mathrm{He}$ atoms smallest red spheres. The red spheres marked "1, 2 " in (a) correspond to octa- 1 and octa- 2 interstitials. (a)

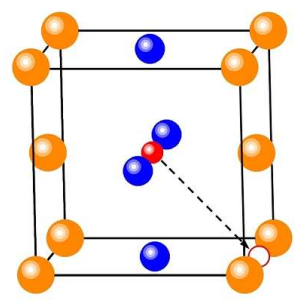

(b)

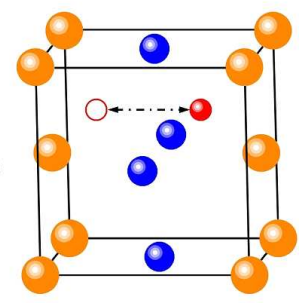

Fig. 5. Migration paths of a He atom in an $\mathrm{Au}_{3} \mathrm{Ag}_{2}$ alloy, (a) from octa-2 to tetra-1, (b) between two neighbor tetra sites. Au atoms are shown by the biggest golden spheres, Ag atoms medium blue spheres. The smallest hollow red sphere represents the initial position of a $\mathrm{He}$ atom, while the smallest solid red sphere the final position, respectively. The arrow indicates the direction of the pathway of He.

with the formation energy difference between octa-2 and octa-1 in $\mathrm{AuAg}$ alloy. If an octa-1 He atom migrates to octa-2 site, there are no migration energy barriers, which means that a less stable octa-1 He atom will automatically migrate to the most stable octa-2 position. In fact, the octa- 1 site is saddle point of the migration path of octa-2-octa-1-octa-2. The migration energy barrier from octa- 2 to octa- 1 is $0.52 \mathrm{eV}$, which is higher than $0.32 \mathrm{eV}$ of the migration energy barrier from octa- 1 to nearest octa-1 via tetra-3 site in $\mathrm{Au}_{3} \mathrm{Ag}_{2}$ alloy. These results obtained above suggest that an octa- 2 He atom thermal migration to a nearest octa-2 via octa-1 is more difficult at room temperature, similar to that of the migration of octa-1-tetra-3-octa-1 in $\mathrm{Au}_{3} \mathrm{Ag}_{2}$ alloy.

From the red curve of Fig. 6, it can be seen that the migration energy of He atom migrating between two nearest tetra sites is $0.41 \mathrm{eV}$, which is lower than the $0.52 \mathrm{eV}$, the migration energy barrier from octa- 2 to octa- 1 in $\mathrm{AuAg}$ alloy. It means that He atoms migration between two nearest tetra sites is easier than octa- 2 to octa- 1 . On the basis of the above analysis, He atoms prefer to occupy octa-2 interstitials and it is almost impossible to thermally migrate from octa- 2 to nearest octa- 2 via octa- 1 position at room temperature. The tetra interstitials $\mathrm{He}$ atoms may migrate between nearest tetra sites. 


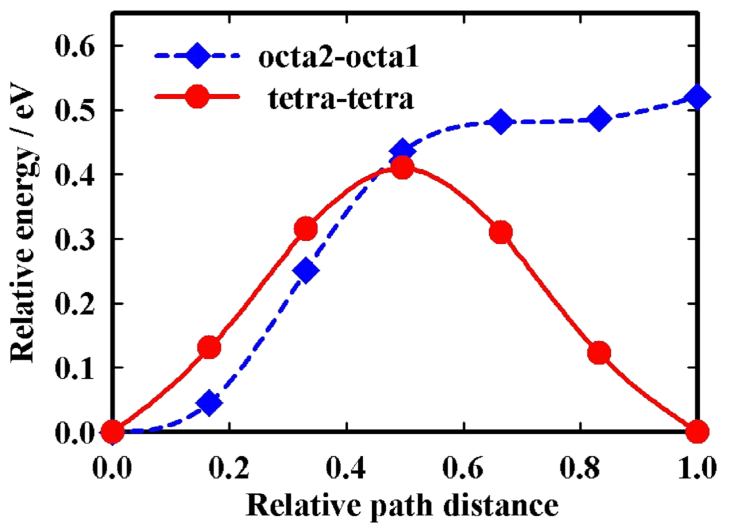

Fig. 6. Migration energy paths of a $\mathrm{He}$ atom from octa- 2 to tetra- 1 and between two neighbor tetra sites in an $\mathrm{AuAg}$ alloy, and the corresponding migration paths shown in Fig. 5.

\section{Conclusion}

The migration mechanisms of $\mathrm{He}$ in $\mathrm{Au}_{3} \mathrm{Ag}_{2}$ and $\mathrm{AuAg}$ alloys are investigated by using CI-NEB. One possible migration mechanism in $\mathrm{Au}_{3} \mathrm{Ag}_{2}$ alloy and two in $\mathrm{AuAg}$ alloy are considered, and the results show that He atoms prefer to occupy the most stable sites, and almost it is impossible for He to migrate from the most stable sites to nearest most stable sites via second stable position at room temperature, similar to that of the He defects migrating in hcp-Sc by the path of tetra-octa-tetra. The tetra interstitials $\mathrm{He}$ atoms in $\mathrm{AuAg}$ alloy may migrate between nearest tetra sites. Comparing the migration of He defects in the two alloys, we found that the properties of migration energy and relative stability of He atoms probably slightly depends on the mass-density of $\mathrm{Au}-\mathrm{Ag}$ alloys. Because the mechanical properties of the $\mathrm{Au}-\mathrm{Ag}$ alloys need to be studied in both theory and experiment, we can only make a conclusion that the $\mathrm{Au}_{3} \mathrm{Ag}_{2}$ alloy may be a suitable surrogate.

\section{Acknowledgments}

Z.Q. Zhu and Y.L. Zhang are grateful for the support by the Youth Natural Science Foundation of Zhoukou Normal University (Grant No. zknuc0207).

\section{References}

[1] A.J. Schwartz, W.G. Wolfer, J. Comp.-Aided Mater. Des. 3, 14 (2007).

[2] W.G. Wolfer, Los Alamos Sci. 26, 274 (2000).

[3] V. Dremov, P. Sapozhnikov, A. Kutepov, Phys. Rev. B. 77, 224306 (2008).

[4] B.Y. Ao, X.L. Wang, W.Y. Hu, J.Y. Yang, J.X. Xia, J. Alloys Comp. 300, 444 (2007).

[5] A. A. Gnidenko, V.G. Zavodinsky, A. Misiuk, J. BakMisiuk, Acta Phys. Pol. A 109, 353 (2006).

[6] B.Y. Ao, P.H. Chen, P. Shi, X.L. Wang, W.Y. Hu, L. Wang, Commun. Comput. Phys. 11, 1205 (2012).

[7] O.J. Wick, Plutonium Handbook - A Guide to the Technology, Gordon and Breach, New York 1967, p. 63.

[8] D.A. Young, Phase Diagrams of the Elements, University of California Press, Berkeley 1991, p. 224.

[9] J. Wong, M. Wall, A.J. Schwartz, R. Xu, M. Holt, H. Hong, P. Zschack, T.C. Chiang, Appl. Phys. Lett. 19, 84 (2004).

[10] N. Baclet, B. Oudot, R. Grynszpan, L. Jolly, B. Ravat, P. Faure, L. Berlu, G. Jomard, J. Alloys Comp. 305, 444 (2007).

[11] M.F. Stevens, T. Zocco, R. Albers, J.D. Becker, K. Walter, B. Cort, D. Paisley, M. Nastasi, Final Report: Fundamental and Applied Studies of Helium Ingrowth and ageing in Plutonium, LA-UR-98-2606, 1998.

[12] T. Thomé, R.I. Grynszpan, Radiat. Eff. Deff. S. 6 , 161 (2006).

[13] Z.Q. Zhu, L. Yang, J.L. Nie, S.M. Peng, X.G. Long, X.S. Zhou, X.T. Zu, F. Gao, J. Alloys Comp. 5, 557 (2013).

[14] R.C. Chen, L. Yang, Y.Y. Dai, Z.Q. Zhu, S.M. Peng, X.G. Long, F. Gao, X.T. Zu, Chin. Phys. B 5, 056601 (2012).

[15] G. Henkelman, B.P. Uberuaga, H. Jónsson, J. Chem. Phys. 113, 9901 (2000).

[16] L. Yang, X.T. Zu, F. Gao, Physica B Condens. Matter 403, 2719 (2008).

[17] L. Yang, S.M. Peng, X.G. Long, F. Gao, H.L. Heinisch, R.J. Kurtz, X.T. Zu, J. Phys. Condens. Matter. 23, 035701 (2011).

[18] M.D. Whitmore, J. Phys. F Met. Phys. 6, 1259 (1976). 